\title{
Safety and Efficacy of Ranibizumab and Luseogliflozin Combination Therapy in Patients with Diabetic Macular Edema: Protocol for a Multicenter, Open- Label Randomized Controlled Trial
}

\author{
Ryoichi Ishibashi (D) - Yoko Takatsuna - Masaya Koshizaka · Tomoaki Tatsumi · Sho Takahashi • \\ Kengo Nagashima - Noriko Asaumi · Miyuki Arai · Fumio Shimada - Kaori Tachibana • \\ Yoshihiro Watanabe - Ko Ishikawa · Akiko Hoshino · Kyohei Yamamoto · Mariko Kubota-Taniai • \\ Takafumi Mayama · Shuichi Yamamoto $\cdot$ Koutaro Yokote
}

Received: May 15, 2020 / Published online: June 15, 2020

(C) The Author(s) 2020

\section{ABSTRACT}

Introduction: Diabetic macular edema (DME) threatens daily life activities such as reading and driving and reduces the patients' quality-of-life. Recently, anti-vascular endothelial growth factor (VEGF) agents have become a first-line

Digital Features To view digital features for this article go to https://doi.org/10.6084/m9.figshare.12403526.

R. Ishibashi $(\square)$

Department of Medicine, Division of Diabetes, Endocrinology and Metabolism, Kimitsu Chuo Hospital, 1010 Sakurai, Kisarazu, Chiba 292-8535, Japan

e-mail: ishibashi-cib@umin.net

R. Ishibashi · M. Koshizaka · K. Yokote

Department of Endocrinology, Hematology and Gerontology, Chiba University Graduate School of Medicine, 1-8-1 Inohana, Chuo-ku, Chiba, Chiba 260-8677, Japan

\section{Y. Takatsuna}

Department of Ophthalmology, Chiba Rosai Hospital, 2-16 Tatsumidai-Higashi, Ichihara, Chiba 290-0003, Japan

Y. Takatsuna - T. Tatsumi $\cdot$ S. Yamamoto Department of Ophthalmology and Vision Science, Chiba University Graduate School of Medicine, 1-81 Inohana, Chuo-ku, Chiba, Chiba 260-8677, Japan

M. Koshizaka $\cdot$ K. Yokote

Department of Diabetes, Metabolism and

Endocrinology, Chiba University Hospital, 1-8-1

Inohana, Chuo-ku, Chiba, Chiba 260-8677, Japan therapy in DME. However, therapy with antiVEGF agents has several problems: repeated invasive injections are required; medical costs are high; and a certain proportion of patients with DME are resistant to treatment with antiVEGF agents. While sodium-glucose co-transporter 2 (SGLT2) inhibitors have been widely used for the treatment of type 2 diabetes mellitus (T2DM), the effects of a combination

\section{S. Takahashi}

Clinical Research Support Center, The Jikei

University School of Medicine, 3-25-8 NishiShimbashi, Minato-ku, Tokyo 105-8461, Japan

\section{K. Nagashima}

Research Center for Medical and Health Data Science, The Institute of Statistical Mathematics, 103 Midori-cho, Tachikawa, Tokyo 190-8562, Japan

N. Asaumi

Department of Ophthalmology, Kimitsu Chuo Hospital, 1010 Sakurai, Kisarazu, Chiba 292-8535, Japan

\section{Arai}

Department of Ophthalmology, National Hospital Organization Chiba Medical Center, Tsubakimori 4-1-2, Chuo-ku, Chiba, Chiba 260-8606, Japan

F. Shimada

Department of Diabetes and Metabolism, National Hospital Organization Chiba Medical Center, Tsubakimori 4-1-2, Chuo-ku, Chiba, Chiba 2608606, Japan 
therapy with anti-VEGF agent and SGLT2 inhibitor on DME are not yet known.

Methods: This study enrolls subjects with T2DM and DME, randomizes them into either a study agent treatment group (treated with ranibizumab as anti-VEGF agent and luseogliflozin as SGLT2 inhibitor) or a control group (treated with ranibizumab and glimepiride), and observes the subjects for 52 weeks after initiation of treatment. Planned outcomes: The primary endpoint is intergroup difference in the number of intravitreal anti-VEGF injections to the study eye from baseline to week 48 . Secondary and exploratory endpoints include safety and ophthalmologic and internal medical clinical parameters.

Registration: This study is registered at the University Hospital Medical Information Network Clinical Trial Registry (UMIN000033961) and Japan Registry of Clinical Trials (jRCTs031180210).

Keywords: Anti-vascular endothelial growth factor agent; Central retinal thickness; Diabetic macular edema; Sodium-glucose transporter 2 inhibitor; Optical coherence tomography

K. Tachibana

Department of Diabetes, Metabolic Diseases, Endocrinology, Japanese Red Cross Narita Hospital, 90-1, Iida-cho, Narita, Chiba 286-8523, Japan

Y. Watanabe

Department of Ophthalmology, Japanese Red Cross Narita Hospital, 90-1, Iida-cho, Narita, Chiba 2868523, Japan

\section{K. Ishikawa}

Department of Internal Medicine, Chiba Rosai

Hospital, Tatsumidai-Higashi 2-16, Ichihara, Chiba 290-0003, Japan

\section{A. Hoshino}

Department of Ophthalmology, Chiba Aoba Municipal Hospital, Aoba-cho 1273-2, Chuo-ku, Chiba, Chiba 260-0852, Japan

\section{Key Summary Points}

\section{Why carry out this study?}

There are unmet needs in anti-vascular endothelial growth factor (VEGF) agents for diabetic macular edema (DME); they require repeated invasive injection and have high medical costs.

Several reports have suggested that sodium-glucose co-transporter 2 (SGLT2) inhibitors have some possibility of improving DME; however, the effect of combination therapy with anti-VEGF agent and SGLT2 inhibitor on DME is not yet revealed.

The aim of this study is to determine whether the combination therapy with anti-VEGF agent and SGLT2 inhibitor for DME reduces the number of anti-VEGF agent injections.

\section{What was learned from the study?}

This study could show a new option for treatment of DME.
K. Yamamoto

Department of Internal Medicine, Chiba Aoba Municipal Hospital, Aoba-cho 1273-2, Chuo-ku, Chiba, Chiba 260-0852, Japan

\section{Kubota-Taniai}

Department of Ophthalmology, Chiba Kaihin Municipal Hospital, Isobe 3-31-1, Mihama-ku, Chiba, Chiba 261-0012, Japan

\section{T. Mayama}

Department of Internal Medicine, Chiba Kaihin Municipal Hospital, Isobe 3-31-1, Mihama-ku, Chiba, Chiba 261-0012, Japan 


\section{INTRODUCTION}

The prevalence of type 2 diabetes mellitus (T2DM) is increasing worldwide, and according to the International Diabetes Federation, the number of patients with T2DM globally was 382 million in 2013 [1] and is speculated to reach to 592 million in 2030 [2]. A national survey in Japan reported that the possible prevalence of diabetes mellitus (DM) in Japan was 6.9 million in 1997, and this was expected to increase to 10 million by 2016 [3]. Chronic hyperglycemia in patients with DM causes microvascular and macrovascular complications such as diabetic nephropathy, retinopathy, diabetic neuropathy, ischemic heart disease, cerebral stroke, and foot gangrene, all of which can affect the patients' prognosis and qualityof-life (QOL).

The visual acuity reduction or visual loss associated with diabetic retinopathy can be attenuated or prevented by recent advances in the treatment of DM, including panretinal photocoagulation and vitreous surgery [4-6]. However, diabetic macular edema (DME) remains a significant healthcare issue, since DME interferes with everyday activities such as reading and driving, thus reducing the patients' QOL. A recent molecular biological study revealed that vascular endothelial growth factor (VEGF) was highly expressed in the vitreous of patients with DME, and intravitreal injection of anti-VEGF agents has been established as a firstline therapy for DME [7]. However, anti-VEGF therapies have several problems: repeated injections are required; medical costs are high; and a certain proportion of patients with DME do not respond to anti-VEGF agents [8]. In addition, the pathology of DME is highly complex, involving not only high local expression of VEGF in vitreous and traction of the vitreous membrane but also additional systemic factors, including an increase of hydrostatic pressure and a reduction of plasma osmolality. It has been reported that fluid retention in advanced diabetic nephropathy exacerbates DME, as does the use of pioglitazone, which causes systemic edema $[9,10]$. Together, these observations emphasize the importance of treating patients with T2DM and DME with a full consideration of their pathological conditions in a collaboration between internists and ophthalmologists.

Recently, a new type of oral hypoglycemic agent, sodium-glucose co-transporter 2 (SGLT2) inhibitors, has been launched, and it has already been widely used in the treatment of T2DM. SGLT2 contributes to glucose reabsorption in the proximal renal tubule. Although plasma glucose is filtered by glomeruli in the kidney and excreted into glomerular filtrate, approximately $90 \%$ of glucose (approximately $180 \mathrm{~g} /$ day) in glomerular filtrate is reabsorbed by the proximal renal tubule and returned to the blood. SGLT2 in the proximal renal tubule co-transports glucose and sodium from the glomerular filtrate back into the blood, and SGLT2 inhibitors interfere with glucose reabsorption such that glucose is excreted into urine, thus lowering plasma glucose levels [11]. SGLT2 has also been reported to be highly expressed in the proximal renal tubules of patients with T2DM [12]. SGLT2 inhibitors have several advantages for T2DM treatment. SGLT2 inhibitors lower the plasma glucose in an insulin-independent manner, and therefore the risk of hypoglycemia is low [13]. In addition, recent large-scale clinical trials have demonstrated that SGLT2 inhibitors prevent all causes of mortality, heart failure, and the progression of renal impairment [14, 15]. The mode of action of these effects is inferred to be independent of glycemic control, and to include the effect of natriuresis. Other effects of SGLT2 inhibitors, such as attenuating the burden on pancreatic beta cells, restoring insulin secretory function, and ameliorating glucotoxicity were also expected [16].

Several studies have also demonstrated the favorable effect of SGLT2 inhibitors on DME, including a case report of patients with T2DM and ophthalmologic treatment-resistant DME which was rapidly improved by administration of SGLT2 inhibitors $[17,18]$ and a clinical trial which demonstrated that dapagliflozin improved DME compared with placebo [19]. However, negative results suggesting that SGLT2 inhibitors do not improve DME have also been reported [20]. Therefore, a consensus on the effects of SGLT2 inhibitors on DME has 
not yet been established. The aforementioned studies involved retrospective studies with only a few cases or randomized controlled trials of a small cohort. In addition, these studies did not use anti-VEGF agents, which are currently the first-line therapy in DME, and the effects of a combination therapy with anti-VEGF agents and SGLT2 inhibitors have not yet been investigated. If administration of SGLT2 inhibitors could enhance the effect of anti-VEGF agents on DME, the burden on patients of invasive treatment with intravitreal injection of anti-VEGF agent could be reduced, as could the high medical costs of anti-VEGF agents. Furthermore, a collaboration between physicians and ophthalmologists could manage T2DM treatment more safely and effectively though considering all diabetic complications.

This aim of this study was to assess the safety and efficacy of combination therapy with antiVEGF agent and SGLT2 inhibitor in patients with T2DM and DME, and to compare the results of combination therapy with those of conventional T2DM treatment (glimepiride) and anti-VEGF agent.

\section{METHODS}

\section{Study Design and Setting}

The "Effectiveness and safety of Combination therapy of ranibizumab and luseOgliflozin in type 2 diabetes with diabetic Macular Edema, a parallel group comparison with the standard Treatment controlled by glimepiride, multicenter, randomized, open-label trial" (COMET Trial) was initiated in April 2018 and is set to finish in September 2023. Subjects were enrolled from April 2018 and enrollment will finish in September 2021. As shown in Fig. 1, subjects who are eligible for anti-VEGF agents are asked to participate in this study, and informed consent is sought prior to the screening test. If subjects meet the eligibility criteria of this study during the screening test, they are enrolled and randomized into a study agent treatment group or a control group.

\section{Sample Size Calculation}

The aim of this study is to enroll 60 subjects (30 for the study agent treatment group and 30 for the control group). Because no previous studies have evaluated the effects of combination therapy with anti-VEGF agents and SGLT2 inhibitors, we referred to the results of a clinical trial of combination therapy with intravitreal anti-VEGF injection and navigated laser photocoagulation (laser + anti-VEGF group) vs monotherapy of intravitreal anti-VEGF injection (anti-VEGF monotherapy group) to calculate the sample size [21], in which the number of intravitreal anti-VEGF injections from baseline to week 48 was $3.9 \pm 1.3$ in the laser +

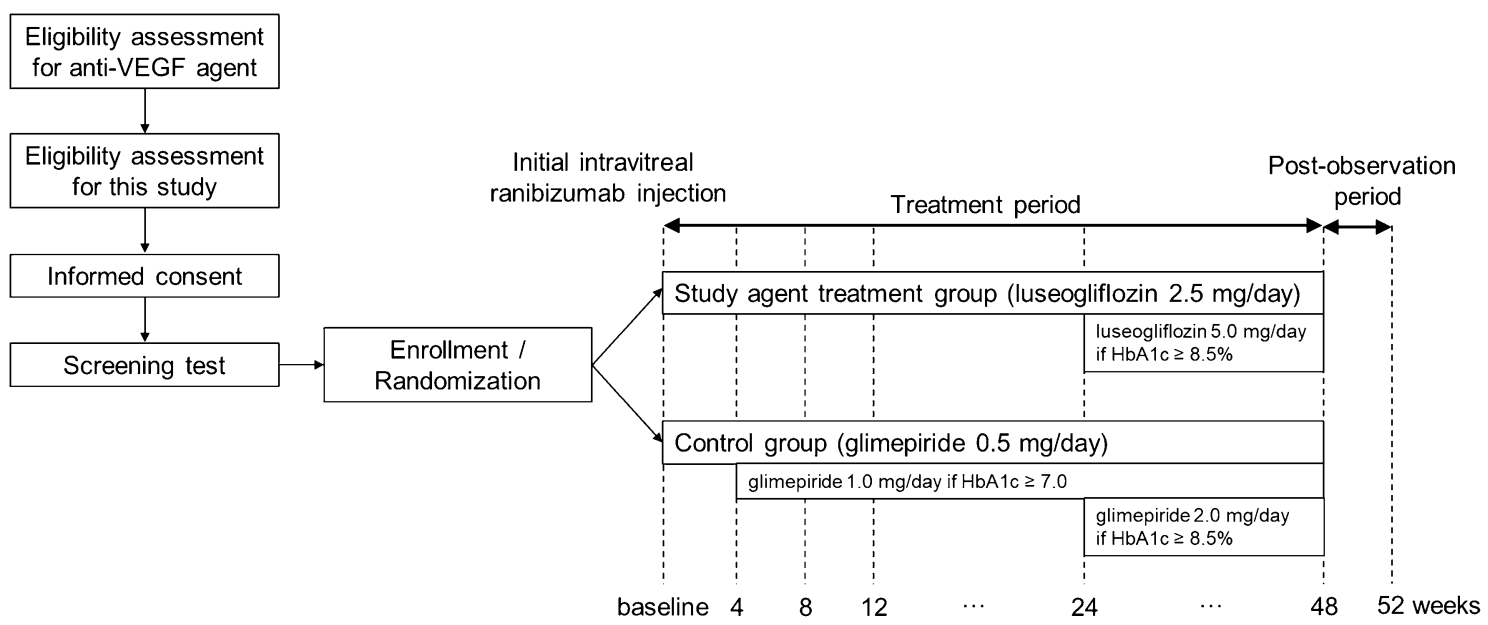

Fig. 1 Flow diagram of study recruitment, randomization, study treatment, and observation 
anti-VEGF group and $6.9 \pm 2.3$ in the antiVEGF monotherapy group. We assumed that the effects in the control group in this study would be comparable to the anti-VEGF monotherapy group of the previous study, and the effects in the study agent treatment group in this study would be slightly less than the laser + anti-VEGF group in the previous study (although the study agent treatment may reduce the overall number of intravitreal antiVEGF injections). We set that superiority is achieved if the intergroup difference in the number of intravitreal anti-VEGF injections was two or more. Assuming that the number of intravitreal anti-VEGF injections from baseline to week 48 was 4.9 in the study agent treatment group and 6.9 in the control group, with a standard deviation of 2.5 , the minimum sample size required to achieve a significance of 0.05 from a two-sided test with a statistical power of $80 \%$ was determined to be 26 subjects for both groups (a total of 52 subjects). To allow for a $10 \%$ dropout rate, the planned enrollment was set at 60 subjects, with 30 in each group.

\section{Eligibility Criteria}

Patients with T2DM and the complication of DME are asked to participate in this study. The detailed inclusion criteria were as follows: (1) patients with T2DM between 20 and 80 years of age at consent; (2) patients with DME which affects the fovea, and with best corrected visual acuity (BCVA) of 0.05 or higher (BCVA converted to logarithm of minimum angle of resolution, $\log \mathrm{MAR}$, of 1.30 or less); (3) patients with a central retinal thickness (CRT) in the study eye of $350 \mu \mathrm{m}$ or more (measured by optical coherence tomography, OCT) at the screening test (if the CRT values of both eyes are $350 \mu \mathrm{m}$ or more, the eye with the thicker retina is defined as the study eye); (4) patients with HbA1c of $6.5 \%$ or higher and less than $12.0 \%$ at the screening test; (5) patients with body mass index (BMI) of $18.5 \mathrm{~kg} / \mathrm{m}^{2}$ or higher at the screening test; (6) patients with estimated glomerular filtration rate (eGFR) of $30 \mathrm{~mL} / \mathrm{min} /$ $1.73 \mathrm{~m}^{2}$ or higher at the screening test; (7) patients for whom DM treatment has not been changed in the 8 weeks preceding the screening test; (8) patients who provide a written consent form to participate in this study after full explanation of the study.

The exclusion criteria were as follows: (1) patients with type $1 \mathrm{DM}$; (2) patients with a history of hypersensitivity against SGLT2 inhibitor or glimepiride; (3) patients with a history of metabolic acidosis, or coma or precoma due to DM or hypoglycemia within the 24 weeks preceding the screening test; (4) patients with severe infections, before or after surgery, or severe trauma which requires insulin treatment; (5) patients with severe renal dysfunction (eGFR $<30 \mathrm{~mL} / \mathrm{min} / 1.73 \mathrm{~m}^{2}$ ), or patients who are undergoing dialysis (including peritoneal dialysis); (6) female patients who are pregnant, possibly pregnant, or plan to be pregnant or breast-feeding; (7) patients with urinary tract infection or dehydration, or patients who are prone to urinary tract infection or dehydration; (8) patients with positive urinary ketone body (urine qualitative of $2+$ or higher); (9) patients with a history of leg amputation due to leg gangrene; (10) patients with a history of bone fracture due to osteoporosis; (11) patients who were treated by thiazolidine, SGLT2 inhibitors, sulfonylurea, or glinide within 8 weeks preceding the screening test; (12) patients who were treated with fibrate or diuretic agents and who changed the usage or dose of them within 8 weeks preceding the screening test; (13) patients who had received systemic administration of steroid within 8 weeks preceding the screening test; (14) patients with a history of cerebrovascular impairment or myocardial infarction within 48 weeks preceding the screening test; (15) patients with poorly controlled hypertension (clinic blood pressure measured in sitting position is $180 \mathrm{mmHg}$ or higher in systolic blood pressure or $100 \mathrm{mmHg}$ or higher in diastolic blood pressure); (16) patients with a history of vitrectomy or scleral buckling; (17) patients with a history of filtration surgery to the study eye for glaucoma treatment, or patients who are expected to require filtration surgery in the future; (18) patients with active proliferative diabetic retinopathy in the study eye; (19) patients with a history of idiopathic or autoimmune uveitis in the study eye; (20) patients with vitreomacular 
traction syndrome or epi-retinal membrane in the study eye (as measured by slit lamp microscope or OCT) which affects central visual acuity; (21) patients with iris neovascularization, vitreous hemorrhage, or traction retinal detachment in the study eye; (22) patients with epi-retinal fibrosis in the study eye, which affects macular area; (23) patients with morphological impairment at the central area of macula lutea in the study eye which can affect the improvement of visual acuity after the disappearance of macular edema (including those with atrophy of retinal pigment epithelium, fibrogenesis or scarring under the retina, severe retina ischemia or structural hard exudate); (24) patients with a history of cataract surgery or other intraocular surgeries to the study eye within 12 weeks preceding the screening test; (25) patients with a history of panretinal laser or macular photocoagulation to the study eye within 12 weeks preceding the screening test; (26) patients with history of yttrium-aluminum-garnet laser posterior capsulotomy to the study eye within 4 weeks preceding the screening test; (27) patients who received steroid to the study eye or the study eye surroundings within 16 weeks preceding the screening test; (28) patients who received angiogenic inhibitor (pegaptanib, bevacizumab, ranibizumab, aflibercept, and others) to any eyes within 12 weeks preceding the screening test; (29) patients in which the transparency of optic media in the study eye is insufficient to obtain fundus or OCT images; (30) patients who received systemic administration of angiogenic inhibitor within 24 weeks preceding the screening test; (31) patients with complications other than diabetic macular edema, which may cause worsening of visual acuity requiring surgical intervention during the study period, or which can affect the study endpoints; (32) patients with any signs of infectious blepharitis, keratitis, scleritis, or conjunctivitis in any eyes; (33) patients with a history of hypersensitivity against anti-VEGF agent or fluorescein; (34) patients who used kallidinogenase or sairei-to (Chinese medicinal herbal drugs) within 8 weeks preceding the screening test; (35) patients with other conditions that the investigator/researcher thinks inappropriate for the study.

\section{Recruitment and Consent}

The informed consent document is offered to subjects who are eligible for anti-VEGF agent to provide a comprehensive explanation of this study. Written consent is then obtained, after which the screening test is conducted to check the eligibility of the subject for this study. Subjects who meet all of the inclusion criteria and do not fail any of exclusion criteria are enrolled in this study.

\section{Random Allocation}

After informed consent is obtained, eligible subjects are randomly assigned in an approximately $1: 1$ ratio to the study agent treatment group or the control group. The randomization sequence was generated using a computer-based dynamic allocation method with a minimization procedure to balance three allocation factors (HbA1c, urinary albumin, and BCVA).

\section{Study Intervention}

All enrolled subjects receive an intravitreal injection of $0.5 \mathrm{mg}$ anti-VEGF agent (ranibizumab) at the start of the study. Within 7 days after the initial intravitreal ranibizumab injection, subjects assigned to the study agent treatment group and the control group begin treatment with either the study agent (luseogliflozin) or control agent (glimepiride), respectively. Luseogliflozin $(2.5 \mathrm{mg})$ is administered to the subjects in the study agent treatment group once daily before or after breakfast. Glimepiride $(0.5 \mathrm{mg})$ is administered to the subjects in the control group once daily before or after breakfast. These luseogliflozin and glimepiride doses are maintained until week 4 . If $\mathrm{HbA} 1 \mathrm{c}$ is $7.0 \%$ or higher at week 4 , the dose of luseogliflozin is maintained in the study agent treatment group, while the dose of glimepiride is increased to $1 \mathrm{mg} /$ day in the control group. If hypoglycemia (symptomatic hypoglycemia or plasma glucose of less than $70 \mathrm{mg} / \mathrm{dL}$ measured 
by self-measurement of blood glucose, SMBG) is observed from baseline to week 4 , glimepiride is not increased. If $\mathrm{HbA} 1 \mathrm{c}$ is $8.5 \%$ or higher at weeks 24-48, the dose of luseogliflozin is increased to $5 \mathrm{mg}$ /day in the study agent treatment group, while the dose of glimepiride is increased to $2 \mathrm{mg}$ /day in the control group. If hypoglycemia is observed during weeks 4 and 24, luseogliflozin and glimepiride are not increased. The dose of luseogliflozin or glimepiride can be reduced if researchers decide that a dose reduction is required because of the occurrence of hypoglycemia or other adverse events. All study subjects are observed every 4 weeks by an ophthalmologist.

Additional intravitreal ranibizumab injections are carried out if the subjects meet the following criteria: (1) CRT measured by OCT is more than $350 \mu \mathrm{m}$; (2) CRT measured by OCT increases more than $100 \mu \mathrm{m}$ from the reference CRT obtained after enrollment to the study; (3) CRT measured by OCT increases relative to the previous observation point, and BCVA converted to $\log$ MAR decreases by 0.2 from the BCVA obtained after enrollment to the study. If the subjects meet any of the aforementioned criteria, ranibizumab is injected at a minimum of 3-week intervals. The combination therapy of ranibizumab and luseogliflozin/ glimepiride is continued to week 48 . From week 48 to 52 (the post-observation period), the ranibizumab and luseogliflozin/glimepiride therapies are both discontinued. Observations are made at the time of screening (for the baseline) and then every 4 weeks until week 52 (after the initial intravitreal ranibizumab injection). Table 1 shows the schedule of assessments performed at each observation point, including mandatory and optional assessments.

\section{Outcomes}

The primary endpoint of this study is an intergroup difference in the number of intravitreal anti-VEGF injections to the study eye from baseline to week 48 . Secondary and exploratory endpoints include ophthalmologic and internal medical clinical parameters. The details of the secondary and exploratory endpoints are summarized in Table 2.

\section{Data Collection}

A case report form is used for data collection. A central registration number is used to identify the subjects for anonymization.

Data collection and management is carried out by third-party entities to avoid bias. Data management is performed by Soiken Inc. Data Management Group (the data center). To manage and ensure quality, the study is monitored by the Soiken Inc. Monitoring Group. For additional data quality management, the principal investigator and Central Committee confirm the progress of the study as necessary through the Soiken Inc. Data Management Group to ensure conformance with the protocol and the Ethical Guidelines for Medical and Health Research Involving Human Subjects (Dec. 22, 2014; Ministry of Education, Culture, Sports, Science and Technology/Ministry of Health, Labour and Welfare) and the Clinical Trials Act (April. 14, 2017; Ministry of Education, Culture, Sports, Science and Technology/ Ministry of Health, Labour and Welfare).

\section{Safety Evaluation}

During this study, the investigators constantly monitor any adverse events (AEs) through regular medical checkups. All related AEs, not only side effects to the drugs but also abnormal clinical laboratory test values and any untoward medical occurrence, are reported and documented. Adverse events are followed up until normalization or recovery to a level not considered to be an adverse event, or until 4 weeks after the end of the pre-observation period or study discontinuation.

\section{Statistical Analyses}

Primary, secondary, and exploratory efficacy endpoints will be analyzed using the full analysis set (FAS), and will include all subjects in each of the intervention groups. However, subjects with a severe protocol violation, such as 
Table 1 Observation items and schedule

\begin{tabular}{|c|c|c|c|c|c|c|c|c|c|c|c|}
\hline \multirow[t]{2}{*}{ Items } & \multirow{2}{*}{$\begin{array}{l}\text { Consenting/ } \\
\text { screening }\end{array}$} & \multicolumn{9}{|c|}{ Treatment period (weeks) } & \multirow{2}{*}{$\begin{array}{l}\text { Post-observation } \\
\text { period } \\
52\end{array}$} \\
\hline & & 0 & 4 & 8 & 12 & 16 & 20 & 24 & $\begin{array}{l}28,32,36 \\
40,44\end{array}$ & 48 & \\
\hline \multicolumn{12}{|l|}{ Informed consent } \\
\hline \multicolumn{12}{|l|}{ Subject characteristics } \\
\hline \multicolumn{12}{|l|}{$\begin{array}{l}\text { Concomitant } \\
\text { drug/combination therapy }\end{array}$} \\
\hline $\begin{array}{l}\text { Number of ranibizumab } \\
\text { injections }\end{array}$ & & $\bigcirc$ & $\square$ & $\square$ & $\square$ & $\square$ & $\square$ & $\square$ & $\square$ & $\square$ & \\
\hline \multicolumn{12}{|l|}{ Central retinal thickness } \\
\hline \multicolumn{12}{|l|}{$\begin{array}{l}\text { Subjective or objective } \\
\text { symptom }\end{array}$} \\
\hline \multicolumn{12}{|l|}{ Adverse event } \\
\hline \multicolumn{12}{|l|}{ Visual acuity } \\
\hline Ophthalmoscopy & O & $\triangle$ & & & & & & & & & \\
\hline Fluorescein angiography & $\triangle$ & & & & & & & $\triangle$ & & $\triangle$ & \\
\hline \multicolumn{12}{|l|}{$\begin{array}{l}\text { Optical coherence } \\
\text { tomography }\end{array}$} \\
\hline \multicolumn{12}{|l|}{ Tonometry } \\
\hline \multicolumn{12}{|l|}{ Height } \\
\hline \multicolumn{12}{|l|}{$\begin{array}{l}\text { Body weight/blood } \\
\text { pressure/heart rate }\end{array}$} \\
\hline \multicolumn{12}{|l|}{ Blood tests/urine tests } \\
\hline Urinary L-FABP & $\bigcirc$ & $\triangle$ & & & & & & $\triangle$ & & $\triangle$ & \\
\hline $\begin{array}{l}\text { Urinary albumin/creatinine } \\
\text { ratio }\end{array}$ & $\bigcirc$ & $\triangle$ & & & & & & & & $\bigcirc$ & \\
\hline \multicolumn{12}{|l|}{ Electrocardiogram } \\
\hline \multicolumn{12}{|l|}{ Serum insulin } \\
\hline Body composition & & $\triangle$ & & & & & & & & $\triangle$ & \\
\hline Cardio ankle vascular index & & $\triangle$ & & & & & & & & $\triangle$ & \\
\hline
\end{tabular}

$\bigcirc$ mandatory item, $\triangle$ optional item, $\square$ if applicable

registration without consent, or registration out of the enrollment period, or subjects without any baseline data, will be excluded from the FAS. Analysis using per protocol set (PPS) is to be conducted, if necessary, but will exclude subjects with a protocol violation, such as violation of eligibility criteria, use of prohibited or restricted concomitant drugs, or poor adherence to study agent or control agent (less than $75 \%)$. A safety analysis will be conducted on the 
Table 2 Secondary and exploratory endpoints

Endpoints Items

Efficacy secondary endpoints

Change and percentage change of following items from baseline to each observation point (every 4 weeks up to week 48) and intergroup difference between them
1. Proportion of patients who require additional anti-VEGF intravitreal injections to the study eye after the initial antiVEGF intravitreal injection

2. Proportion of patients who do not require additional antiVEGF intravitreal injections to the study eye between 4 weeks after the initial anti-VEGF intravitreal injection to each observation point (every 4 weeks up to week 48)

3. Number of retinal microaneurysm photocoagulation (MAPC) to retinal arteriolar macroaneurysm of the study eye from baseline to week 48

4. Central retinal thickness (CRT) of the study eye at each observation point measured by optical coherence tomography (OCT)

5. Best corrected visual acuity (BCVA) of the study eye at each observation point converted to logarithm of minimum angle of resolution $(\log M A R)$

6. Presence or absence of hemorrhage at macular area or hard exudate in the study eye at each observation point measured by ophthalmoscopy

7. Presence or absence of active leakage from blood vessels surrounding the macula area of the study eye measured by fluorescein angiography

8. Morphology of macular edema and visual acuity, CRT, number of injections of the study eye at each observation point

9. Body weight, body mass index (BMI), blood pressure, pulse, HbAlc, plasma glucose, eGFR, and hematocrit (Hct) 
Table 2 continued

\section{Endpoints}

Intergroup difference in the change of following items during the post-treatment observation period (4 weeks from week 48 to week 52)

Safety secondary endpoints

Intergroup difference in the frequency and proportion of any adverse events and side effects from baseline to week 48 estimated by MedDRA

Intergroup difference in the occurrence of any adverse events and side effects during the post-treatment observation period ( 4 weeks from week 48 to week 52)

Exploratory endpoints

Change, percentage change, and intergroup difference of following items from baseline to week 24 and week 48

\section{Items}

1. CRT of the study eye measured by OCT

2. BCVA of the study eye converted to logMAR

3. Presence or absence of hemorrhage at macular area or hard exudate in the study eye

4. Presence or absence of active leakage from blood vessels surrounding the macula area in the study eye measured by fluorescein angiography

5. Visual acuity and CRT of the study eye stratified by morphology of macular edema

6. Body weight, BMI, blood pressure, pulse, HbAlc, plasma glucose, eGFR, and Hct

- Insulin and calculated homeostasis model assessment insulin resistance (HOMA-R), homeostatic model assessment beta cell function (HOMA- $\beta$ )

- C-peptide and calculated C-peptide index (CPI)

- Urinary albumin/creatinine ratio

- Biomarker of heart failure (brain natriuretic peptide, BNP)

- Biomarker of kidney and oxidative stress (urinary liver fatty acid-binding protein, L-FABP)

(To be completed only in patients where these items can be measured)

- Cardio ankle vascular index (CAVI)

- Total body water, intracellular water, extracellular water, lean body mass, muscle mass, bone mineral, body fat percentage, and basal metabolism measured by body composition meter (bioelectrical impedance method) 
Table 2 continued

Endpoints
Correlation between intergroup difference in change or
percentage change of following items from baseline to
week 24 and week 48 and primary and secondary
endpoints

\section{Items}

- Body weight, BMI, blood pressure, pulse, HbAlc, plasma glucose, eGFR, and Hct

- Aspartate aminotransferase (AST), alanine aminotransferase (ALT), gamma-glutamyl transpeptidase $(\gamma$-GTP), uric acid (UA), total cholesterol (TC), triglyceride (TG), high-density lipoprotein cholesterol (HDL-C), low-density lipoprotein cholesterol (LDL-C/ indirect measurement), total bilirubins (T-BIL), blood urea nitrogen (BUN), creatinine (Cre), insulin and calculated HOMA-R and HOMA- $\beta$, C-peptide and calculated CPI, urinary albumin/creatinine ratio, BNP, and urinary L-FABP

(To be completed only in patients where these items can be measured)

- CAVI

- Total body water, intracellular water, extracellular water, lean body mass, muscle mass, bone mineral, body fat percentage, and basal metabolism measured by body composition meter (bioelectrical impedance method)

If patients have macular edema in the fellow eye to which anti-VEGF intravitreal injections are not performed, observe same items of primary and secondary endpoints in the fellow eye

safety analysis population, which will include all subjects in each of the intervention groups who received at least one dose of study agent or control agent.

Subject characteristics at baseline will be presented as frequencies and proportions for categorical data, and summary statistics (number of subjects, mean, standard deviation, minimum, median, and maximum) for continuous data. Subject characteristics will then be compared using Pearson's chi-square test or Fisher's exact test for categorical endpoints, and the Student's $t$ test for continuous variables.

The primary endpoint of this study is intergroup difference in the number of intravitreal anti-VEGF injections to the study eye from baseline to week 48 . Intergroup comparison will be performed by analysis of covariance (ANCOVA) with the allocation factors (HbA1c, urinary albumin, and BCVA), and summary statistics and confidence intervals will be calculated. Analysis of secondary endpoints will be performed according to the statistical analysis plan, which will be developed before database lock. Adverse events will be evaluated during the safety analysis. The frequencies of adverse events will be compared using Fisher's exact test. A two-sided $p$ value of less than 0.05 will be considered statistically significant and a twosided 95\% confidence interval will be calculated. The statistical analysis plan will be finalized prior to database lock. All statistical analysis will be conducted by independent biostatisticians. 


\section{Patient and Public Involvement}

No patients nor the general public will be involved in the planning, execution, analysis, and evaluation of the study.

\section{STRENGTHS AND LIMITATIONS OF THE STUDY}

This is the first randomized controlled trial to compare the combination therapy of anti-VEGF agent and SGLT2 inhibitor with the combination therapy of anti-VEGF agent and glimepiride. Compared with glimepiride, SGLT2 inhibitors have a low risk of hypoglycemia [22]. In addition to improving glycemic control, SGLT2 inhibitors have several favorable effects, including reduced risk of cardiovascular and renal diseases $[14,15,23]$. If the SGLT2 inhibitor also demonstrates a retinoprotective effect, it may contribute to new strategies for the treatment of T2DM-associated diabetic retinopathy. The combination therapy of SGLT2 inhibitor with anti-VEGF agent may also contribute to reduced medical costs for the treatment of DME. The drug price of luseogliflozin $(2.5 \mathrm{mg})$ is 180.0 yen/day, whereas that of ranibizumab is 160,698 yen/injection (as of March 2020). The combination therapy of SGLT2 inhibitor and anti-VEGF agent could substantially reduce the economic burden on patients and on the national health insurance system. This combination therapy could also decrease the burden experienced by patients receiving an invasive injection of intravitreal anti-VEGF agent.

We have identified several limitations of our study. The primary limitation is its exploratory nature. Because the effects of the combination therapy of SGLT2 inhibitor with anti-VEGF agent have not been previously evaluated, the sample size calculation was based on the results of an earlier combination therapy involving SGLT2 inhibitor and navigated laser photocoagulation [21]. A second limitation is that this study is conducted only in clinical institutions in Japan, and all recruited subjects are expected to be Japanese, although the eligibility criteria in this study does not exclude non-Japanese ethnicity. This constraint may limit the generalizability of this study.

\section{Ethics and Dissemination}

This study and its protocols were first approved by the institutional review board of each participating institution, according to the Ethical Guidelines for Medical and Health Research Involving Human Subjects issued by the Ministry of Health, Labour and Welfare in Japan. Following publication of the Clinical Trials Act in April 2017, this study and its protocols were again inspected and approved by the Chiba University Certified Clinical Research Review Board, which had obtained certification from the Minister of Health, Labour and Welfare in Japan. The study is conducted in accordance with the Declaration of Helsinki, Ethical Guidelines for Medical and Health Research Involving Human Subjects issued by the Ministry of Health, Labour and Welfare in Japan, the Clinical Trials Act, and other current legal regulations in Japan. Written informed consent was obtained from all subjects after full explanation of this study. Subjects' identities are concealed by using the central registration number. The results of this study will be disseminated at medical conferences and in journal publications.

\section{ACKNOWLEDGEMENTS}

We thank all the participants of this study. The authors also thank all the clinical staff for their assistance in the execution of the study, and Soiken Inc. for their technical assistance in the launch and execution of the study.

Funding. This study and the journals rapid service fee is financially supported by Taisho Pharmaceutical Co., Ltd. Taisho Pharmaceutical Co., Ltd. is not involved in this study, including the planning, execution, data management, statistical analysis, evaluation, or write-up.

Authorship. All named authors meet the International Committee of Medical Journal 
Editors (ICMJE) criteria for authorship for this article, take responsibility for the integrity of the work as a whole, and have given their approval for this version to be published.

Authorship Contributions. RI and YT contributed to the conception of the study. RI, YT, $\mathrm{MK}, \mathrm{TT}, \mathrm{ST}$, and KN contributed to the design of the study. SY and KY contributed to and supervised the conception and design of the study. RI, YT, MK, TT, NA, MA, FS, KT, YW, KI, $\mathrm{AH}, \mathrm{KY}, \mathrm{MK}$, and TM contributed to enrollment and acquisition of data. RI and MK drafted and revised the protocol.

Disclosures. Ryoichi Ishibashi has received research funds from Astellas Pharma Inc., Amgen Astellas BioPharma K.K., Daiichi Sankyo Co. Ltd., Taisho Pharmaceutical Co. Ltd., Takeda Pharmaceutical Co., Ltd., MSD K.K., Boehringer Ingelheim Pharmaceuticals, Inc., and Mitsubishi Tanabe Pharma Corporation. Shuichi Yamamoto has received lecture fee from Alcon Pharmaceuticals Ltd., research fund from Bayer Yakuhin Ltd., and scholarship contribution from Alcon Pharmaceuticals Ltd., Senju Pharmaceutical Co., Ltd., Bayer Yakuhin Ltd., and Santen Pharmaceutical Co., Ltd. Kyohei Yamamoto has received lecture fee from MSD K.K., Mitsubishi Tanabe Pharma Corporation., and Novo Nordisk Pharma Ltd. All funding agencies played no role in the study design, data collection and analysis, decision to publish, or preparation of the manuscript. Yoko Takatsuna, Masaya Koshizaka, Tomoaki Tatsumi, Sho Takahashi, Kengo Nagashima, Noriko Asaumi, Miyuki Arai, Fumio Shimada, Kaori Tachibana, Yoshihiro Watanabe, Ko Ishikawa, Akiko Hoshino, Mariko Kubota-Taniai, Takafumi Mayama and Koutaro Yokote have nothing to disclose.

Compliance with Ethics Guidelines. This study and its protocols were first approved by the institutional review board of each participating institution, according to the Ethical Guidelines for Medical and Health Research Involving Human Subjects issued by the Ministry of Health, Labour and Welfare in Japan. Following publication of the Clinical Trials Act in April 2017, this study and its protocols were again inspected and approved by the Chiba University Certified Clinical Research Review Board, which had obtained certification from the Minister of Health, Labour and Welfare in Japan. The study is conducted in accordance with the Declaration of Helsinki, Ethical Guidelines for Medical and Health Research Involving Human Subjects issued by the Ministry of Health, Labour and Welfare in Japan, the Clinical Trials Act, and other current legal regulations in Japan. Written informed consent was obtained from all subjects after full explanation of this study. The results of this study will be disseminated at medical conferences and in journal publications.

Data Availability. The datasets generated during and/or analyzed during the current study are not publicly available due to the lack of the data sharing statement to the the third party after the end of the study on the study protocol and lack of the approval to the data sharing by the institutional review board of each participating institution or the Chiba University Certified Clinical Research Review Board.

Open Access. This article is licensed under a Creative Commons Attribution-NonCommercial 4.0 International License, which permits any non-commercial use, sharing, adaptation, distribution and reproduction in any medium or format, as long as you give appropriate credit to the original author(s) and the source, provide a link to the Creative Commons licence, and indicate if changes were made. The images or other third party material in this article are included in the article's Creative Commons licence, unless indicated otherwise in a credit line to the material. If material is not included in the article's Creative Commons licence and your intended use is not permitted by statutory regulation or exceeds the permitted use, you will need to obtain permission directly from the copyright holder. To view a copy of this licence, visit http://creativecommons.org/licenses/bync/4.0/. 


\section{REFERENCES}

1. International Diabetes Federation. IDF Diabetes Atlas, 6th ed. Brussels. 2013; Belgium: International Diabetes Federation

2. Saeedi P, Petersohn I, Salpea P, et al. Global and regional diabetes prevalence estimates for 2019 and projections for 2030 and 2045: results from the International Diabetes Federation Diabetes Atlas, 9(th) edition. Diabetes Res Clin Pract. 2019;157: 107843.

3. Ministry of Health, Labour and Welfare of Japan. The National Health and Nutrition Survey, 2016 (in Japanese). https://www.mhlw.go.jp/bunya/kenkou/ kenkou_eiyou_chousa.html. Accessed Oct 31, 2017

4. The Diabetic Retinopathy Study Research Group. Photocoagulation treatment of proliferative diabetic retinopathy. Clinical application of Diabetic Retinopathy Study (DRS) findings, DRS Report Number 8. The Diabetic Retinopathy Study Research Group. Ophthalmology. 1981;88: 583-600.

5. Haller JA, Qin H, Apte RS, et al. Vitrectomy outcomes in eyes with diabetic macular edema and vitreomacular traction. Ophthalmology. 2010;117: 1087-1093.e3.

6. Adelman R, Parnes A, Michalewska Z, Parolini B, Boscher C, Ducournau D. Strategy for the management of diabetic macular edema: the European Vitreo-retinal Society macular edema study. Biomed Res Int. 2015;2015:352487.

7. Funatsu H, Noma H, Mimura T, Eguchi S, Hori S. Association of vitreous inflammatory factors with diabetic macular edema. Ophthalmology. 2009;116:73-9.

8. Bressler NM, Beaulieu WT, Glassman AR, et al. Persistent macular thickening following intravitreous aflibercept, bevacizumab, or ranibizumab for central-involved diabetic macular edema with vision impairment: a secondary analysis of a randomized clinical trial. JAMA Ophthalmol. 2018;136:257-69.

9. Ryan EH Jr, Han DP, Ramsay RC, et al. Diabetic macular edema associated with glitazone use. Retina. 2006;26:562-70.

10. Diep TM, Tsui I. Risk factors associated with diabetic macular edema. Diabetes Res Clin Pract. 2013;100:298-305.

11. Chen LH, Leung PS. Inhibition of the sodium glucose co-transporter-2: its beneficial action and potential combination therapy for type 2 diabetes mellitus. Diabetes Obes Metab. 2013;15:392-402.

12. Rahmoune H, Thompson PW, Ward JM, Smith CD, Hong G, Brown J. Glucose transporters in human renal proximal tubular cells isolated from the urine of patients with non-insulin-dependent diabetes. Diabetes. 2005;54:3427-34.

13. Ghosh RK, Ghosh SM, Chawla S, Jasdanwala SA. SGLT2 inhibitors: a new emerging therapeutic class in the treatment of type 2 diabetes mellitus. J Clin Pharmacol. 2012;52:457-63.

14. Zinman B, Wanner C, Lachin JM, et al. Empagliflozin, cardiovascular outcomes, and mortality in type 2 diabetes. N Engl J Med. 2015;373:2117-288.

15. Neal B, Perkovic V, Mahaffey KW, et al. Canagliflozin and cardiovascular and renal events in type 2 diabetes. N Engl J Med. 2017;377:644-57.

16. Washburn WN, Poucher SM. Differentiating sodium-glucose co-transporter-2 inhibitors in development for the treatment of type 2 diabetes mellitus. Expert Opin Investig Drugs. 2013;22: 463-86.

17. Maeno A. Four cases of diabetic macular edema who were improved by SGLT2 inhibitor. The 23rd annual meeting of the Japanese society of ophthalmic diabetology, W28-1 (in Japanese). 2017

18. Mieno H, Yoneda K, Yamazaki M, Sakai R, Sotozono C, Fukui M. The efficacy of sodium-glucose cotransporter 2 (SGLT2) inhibitors for the treatment of chronic diabetic macular oedema in vitrectomised eyes: a retrospective study. BMJ Open Ophthalmol. 2018;3:e000130.

19. Agrawal P, Gautam A, Sen S, Pursnani N. Effects of dapagliflozin on diabetic macular edema: a prospective, double-blind, randomized, parallel group trial view eposter clinical therapeutics/new technology-pharmacologic treatment of complications. American Diabetes Association's 77th Scientific Sessions, 1311-P-2017. 2017

20. Iijima Y. Effect of SGLT2 inhibitor on 6 cases of diabetic macular edema. The 23rd annual meeting of the Japanese society of ophthalmic diabetology, O1-3 (in Japanese). 2017

21. Liegl R, Langer J, Seidensticker F, et al. Comparative evaluation of combined navigated laser photocoagulation and intravitreal ranibizumab in the treatment of diabetic macular edema. PLoS One. 2014;9:e113981.

22. Muller-Wieland D, Kellerer M, Cypryk K, et al. Efficacy and safety of dapagliflozin or dapagliflozin plus saxagliptin versus glimepiride as add-on to 
metformin in patients with type 2 diabetes. Diabetes Obes Metab. 2018;20:2598-607.
23. Wiviott SD, Raz I, Bonaca MP, et al. Dapagliflozin and cardiovascular outcomes in type 2 diabetes. N Engl J Med. 2019;380:347-57. 